\title{
Thermal-Structural Analysis of Regenerative Cooled Scramjet
}

\author{
Jin Jiang \\ School of Power and Energy, Northwestern Polytechnical University, Xi'an 710072, China \\ Ruoling Zhang \& Feng Wang \\ China Aerodynamics Research and Development Center, Mianyang 621000, China
}

\begin{abstract}
A numerical model is developed to quantitative evaluation of the thermal and structural characteristics of the regenerative cooled scramjet engine. The three-dimensional internal CFD platform for scramjet and the computational program of domestic aviation kerosene properties are incorporated together to develop three dimensional thermal evaluation code. Then the thermal evaluation results from the code can be introduced into the thermal-stress finite element program to complete quantitative structural analysis of engine structure. The results show the temperature and heat flux distribution of the scramjet structure is very uneven. Local plasticity occurs in some areas. The results of this paper can be an valuable reference for the test and design of regenerative cooled scramjet.
\end{abstract}

Keywords: Scramjet, Regenerative cooling, Thermal and structural analysis

\section{Introduction}

The sustained operating conditions of scramjet engines demand designs that include active cooling by the fuel and the use of lightweight materials that withstand extreme heat fluxes under oxidizing conditions. The fuel cools the metallic structure as it passes through very small longitudinal passages. While absorbing the heat from the structure, the fuel is injected into the scramjet combustor and burned at supersonic speed(Mark A S.).

Since the mass flow rate and the heat sink capacity of the cooling liquid hydrocarbon fuel have significant limitation, the cooling channels design is very important. For the liquid rocket, the typical temperature increment of the cooling fuel is $100 \sim 200 \mathrm{~K}$, while for the hydrocarbon fueled scramjet, the typical temperature increment of the cooling fuel is $700 \sim 800 \mathrm{~K}$, besides the fuel endothermic reaction is needed to control. Pratt \& Whitney Space Propulsion has developed prediction tools to achieve quantitative evaluation of the thermal and structural characteristics of the scramjet engine ${ }^{2}$.The scheme that different heat flux areas require different cooling channels was adopted. The comparison between tests and prediction of computer codes were also investigated (Mark A S.)(Wishart D P, Fortin T B, Guinan D, and Modroukas D. 2003). The practice of P\&Windicates two points. One is that the heat flux distribution of scramjet engine exists distinct multidimensional feature, the other is that calculation of the structural and thermal characteristics of the scramjet engine is very important.

In this paper, three-dimensional internal CFD platform for scramjet and the computational program of domestic aviation kerosene properties are incorporated together to develop three dimensional thermal evaluation code. Then the thermal evaluation results from the code can be introduced into the thermal-stress finite element program to complete quantitative structural analysis of engine structure.

\section{Numerical model of the thermal evaluation code}

The wall of scramjet can consist of four layers: a coating, liner, the channel, and the closeout, which can be made of different materials. In the paper, there is no coat and the material of the wall is the stainless steel (1Cr18Ni9Ti). For the numerical procedure, the engine model is subdivided into a number of stations along the combustion gas flow, as shown in Figure 1.The numbering of stations starts with the inlet to the cooling channels and ends at their exit. As for a single cooling channel, because of the symmetry of the configuration, computations are performed for only one cell (see Figure 2). The two sides of the cell are assumed insulated. As shown in Figure 2, a finite difference grid is also superimposed on the aforementioned cell(NARAGHI M H N. 2002).

The integrated form of the heat-conduction equation which has no heat sources, steady and variable thermal conductivity can be used to describe the heat conduction in the wall(Yu Chang-min. 1982)

$$
-\int_{s} k \operatorname{grad} T \cdot \vec{n} d s=0
$$


The difference equations can be derived from an energy balance on a small, finite element fixed in space, where the thermal conductivity is based on the consistent average of the two neighboring nodal temperatures(TAO Wen-quan. 2001), the conductivity is a function of temperature. The finite difference equation for a node (see Figure 3) located in the middle of a material is given by:

$$
\begin{aligned}
& \frac{2 k_{i+1, j, n} k_{i, j, n}}{k_{i+1, j, n}+k_{i, j, n}} \Delta y\left(\frac{1}{2}\left(\Delta S_{i, j}^{n-1, n}+\Delta S_{i, j}^{n, n+1}\right)\right) \frac{T_{i, j, n}-T_{i+1, j, n}}{\Delta x} \\
& +\frac{2 k_{i, j-1, n} k_{i, j, n}}{k_{i, j-1, n}+k_{i, j, n}} \Delta x\left(\frac{1}{2}\left(\Delta S_{i, j}^{n-1, n}+\Delta S_{i, j}^{n, n+1}\right)\right) \frac{T_{i, j, n}-T_{i, j-1, n}}{\Delta y} \\
& +\frac{2 k_{i-1, j, n} k_{i, j, n}}{k_{i-1, j, n}+k_{i, j, n}} \Delta y\left(\frac{1}{2}\left(\Delta S_{i, j}^{n-1, n}+\Delta S_{i, j}^{n, n+1}\right)\right) \frac{T_{i, j, n}-T_{i-1, j, n}}{\Delta x} \\
& +\frac{2 k_{i, j+1, n} k_{i, j, n}}{k_{i, j+1, n}+k_{i, j, n}} \Delta x\left(\frac{1}{2}\left(\Delta S_{i, j}^{n-1, n}+\Delta S_{i, j}^{n, n+1}\right)\right) \frac{T_{i, j, n}-T_{i, j+1, n}}{\Delta y} \\
& +\frac{2 k_{i, j, n} k_{i, j, n+1}}{k_{i, j, n}+k_{i, j, n+1}}\left(\frac{(\Delta x \Delta y)_{n+1}+(\Delta x \Delta y)_{n}}{2}\right) \frac{T_{i, j, n}-T_{i, j, n+1}}{\Delta S_{i, j}^{n, n+1}} \\
& +\frac{2 k_{i, j, n} k_{i, j, n-1}}{k_{i, j, n}+k_{i, j, n-1}}\left(\frac{(\Delta x \Delta y)_{n}+(\Delta x \Delta y)_{n-1}}{2}\right) \frac{T_{i, j, n}-T_{i, j, n-1}}{\Delta S_{i, j}^{n, n-1}}=0
\end{aligned}
$$

The above formula can be ordered as:

$$
T_{i, j, n}^{l}=\frac{\frac{T_{i+1, j, n}^{l-1}}{R_{1}}+\frac{T_{i, j-1, n}^{l-1}}{R_{2}}+\frac{T_{i-1, j, n}^{l-1}}{R_{3}}+\frac{T_{i, j+1, n}^{l-1}}{R_{4}}+\frac{T_{i, j, n+1}}{R_{5}}+\frac{T_{i, j, n-1}}{R_{6}}}{\frac{1}{R_{1}}+\frac{1}{R_{2}}+\frac{1}{R_{3}}+\frac{1}{R_{4}}+\frac{1}{R_{5}}+\frac{1}{R_{6}}}
$$

where $R_{1} \sim R_{6}$ are resistances between node $i, j, n$ and its six neighboring nodes(NARAGHI M H N. 2002).

Similar equations are derived for boundary nodes depending on the boundary conditions. There are three types: convection between the hot gas and the wall, convection between the coolant and the wall, and the relevant outside surface boundary condition. The outside surface boundary condition can be natural convection such as arc heater test, forced convection such as aerodynamic heating during the flight and insulated. The determination of the pertinent properties for the hot gas and coolant can be found in the later development.

Three dimensional thermal evaluation numerical methods can be adopted for each channel. The temperature of each node is function of the temperatures of neighboring nodes (namely four neighboring nodes at the same station and two nodes for the neighboring stations).Along the axial march the temperatures of the latter station is from the previous march (for the first march the initial temperature is assumed). For each station, the Gauss-Siedel iteration is performed to obtain the convergenttemperature distributions along the other two directions at each station. Then the calculations march axially. Once the results of the last station converged, the results of this march are compared with those of the previous march. If the relative difference between the results of two consecutive marches is less than the axial convergence criterion, the program stops, otherwise it continues its axial marches until convergence is achieved(NARAGHI M H N. 2002).

The Eckert reference enthalpy method(HEISER W H, PRATT D T. 1994) can be adopted to ascertain the hot gas side heat flux. The subscript $\mathrm{G}$ denotes combustion gas, the subscript $\mathrm{X}$ denotes the reference condition, the subscript $n$ denotes the ordinal number of stations, the subscript $\mathrm{W}$ denotes the wall, the subscript A denotes the adiabatic conditions, the subscript $\mathrm{S}$ denotes the stagnant conditions. The Eckert reference enthalpy of combustion gas for the $\mathrm{n}$ station can be expressed as

$$
h_{G X n}=0.5\left(h_{G S n}+h_{G W n}\right)+0.22\left(h_{G A W n}-h_{G S n}\right)
$$

Where $h_{G X n}$ is the reference enthalpy of the gas side, $h_{G S n}$ is the combustion gas static enthalpy, $h_{G A W n}$ is the gas-side adiabatic wall enthalpy, $h_{G W n}$ is a function of gas-side wall temperature $T_{G W n}$. Firstly $T_{G W n}$ is assumed at the nth station, then the reference temperature can be derived from formula (4), consequently the reference properties of boundary layer can be ascertained. The thermodynamic properties and transport properties can be gained by complex chemical equilibrium compositions formulae(Yang Shunhua. 2006). The gas-side wall temperature $T_{G W_{n}}$ at the nth station is the average of the temperature of gas side nodes at the station.

Estimates of convective heat transfer are commonly based on an equation of the form:

$$
q_{c n}=S t_{G X n} \cdot \rho_{G X n} V_{G S n}\left(h_{G A W n}-h_{G W n}\right)
$$


The proportion of heat transfer byradiation is very small, therefore radiation heat flux is neglected. The heat fluxes acquired can be input directly at the specified station $n$, the heat flux is equably imposed on the nodes of the gas side wall at the $\mathrm{n}$ station.

For the kerosene in the cooling channel, one dimensional analytical NUSSELT equations and empirical pressure models can be used to determine the distribution of the heat transfer and the total pressure within the cooling channel. The heat transfer coefficients between kerosene and coolant side wall can be obtained by the coolant average temperature, furthermore the Sieder-Tate formula can be adopted(Linne D L, Meyer M L, Edwards T, and Eitman D A.):

$$
\frac{h d}{k_{f}}=N u_{f}=0.027 \operatorname{Re}_{f}^{0.8} \operatorname{Pr}_{f}^{1 / 3}\left(\frac{\eta_{f}}{\eta_{w}}\right)^{0.14}
$$

Except $\eta_{w}$ can be determined by the assumed coolant side wall temperature, the other parameters in the formula (6) can be gained by the average temperature of the kerosene. The hydraulic diameter of cooling channel can be used as the characteristic length. Because $\eta_{w}$ (The properties of the kerosene is introduced in the following text) is associated with the coolant side wall temperature, the solution needs iteration. Three corresponding heat transfer coefficients can be estimated which are based on the average temperatures of the lower, upper and side-wall .(see identifiers 1, 2, 3 in Fig.2 ).Three heat transfer coefficients and the average temperature of kerosene can be an input for the coolant side boundary conditions at the $\mathrm{n}$ station.

The heat transfer rate $Q_{c}$ from the coolant side walls to the kerosene can be attained by integrating the heat flux along the coolant side walls, the unit is $\mathrm{W} / \mathrm{m}$. Therefore the coolant temperature increment of neighboring stations can be:

$$
T_{n+1}-T_{n}=Q_{c} \Delta s_{n+1, n} /\left(C_{p} m_{l}\right)
$$

Where $C_{p}$ is the specific heat determined bythe average kerosene temperature of the two neighboring stations, $m_{l}$ is the mass flow rate of kerosene in the cooling channel, $\Delta s_{n+1, n}$ is the distance between two neighboring stations.

\section{The calculation of the kerosene properties}

One way to determine the physio-chemical properties of kerosene is to have the knowledge of its species. The components of one aviation kerosene at normal temperature were measured by two universities in China. One measurement was conducted in Tian Jin University using mass spectrograph, and the other in USTC using the method of single vacuum ultraviolet photon ionization combining the mass spectrum of molecular beam.

According to the two measurements, nearly 100 species were detected, mainly C8-C13, among which the most abundant component had a molar concentration of $10.6 \%$. Considering the convenience of calculation, one surrogate model for aviation kerosene should be found complying with at least the following two principles. Firstly, the surrogate should have only a few components, less than 5 for example. Secondly, the surrogate should have nearly the same physical properties as the original kerosene. In principle, the surrogate should also have similar chemical behaviors as the original kerosene. In this paper the surrogate is consisted of $79 \%$ of n-decane, $13 \%$ of 1,1,2-trimethylcyclohexane, and $8 \%$ of $\mathrm{n}$-hexylbenzene, and its critical point is $\mathrm{Tc}=622.5 \mathrm{~K}$, $\mathrm{pc}=2.198 \mathrm{MPa}$. The surrogate has nearly the same physical properties as the original aviation kerosene.

The pressure of the kerosene flowing inside the cooling channel of the regenerative cooled scramjet is above critical pressure (about 2.1MPa 2.5MPa). The fuel can't boil, whereas the fuel temperature can exceed the critical value. Bogus boiling can occur, thenthe efficiency of the heat transfer will depress. The properties of the aviation kerosene can be obtained by one computer program for predicting the thermodynamic and transport properties of hydrocarbon mixtures. The program can also obtain the properties of Bogus boiling fuel, therefore heat transfer depravation aroused by bogus boiling phenomena can be resolved.

\section{Scramjet model with cooling channels}

The height of the isolated section entrance for the scramjet model is $30 \mathrm{~mm}$ and the width is $100 \mathrm{~mm}$. Cooling channels are disposed on the top, bottom and side wall along the combustion gas flow direction from the isolated entrance to the nozzle entrance (See Fig. 4). The height of cooling channel is $3 \mathrm{~mm}$, the width is $2 \mathrm{~mm}$, the liner thickness is $1 \mathrm{~mm}$, the closeout thickness is $3 \mathrm{~mm}$. The fin thickness is $1.5 \mathrm{~mm}$ on the most locations. But after the second cavity, the fin thickness of side wall range from $1.5 \mathrm{~mm}$ to $2.1473 \mathrm{~mm}$. There are 28 cooling channels respectively on the top and bottom wall. There are 5 segments for the side wall, the amount of the cooling 
channel for the five segments (along the combustion gas flow direction) are 9, 16, 12, 18 and 13.

\section{The calculation of the combustion gas flow properties}

Since the scramjet model has the cavities, through the engine flow path the flow field is not uniform along the circumference. Therefore three dimensional CFD result by CARDC-AHL can be inputs for the thermal evaluation.

The CFD group of Airbreathing Hypersonic Laboratory (AHL) of CARDC, CARDC-AHL, has been carrying on the CFD research of internal combustion flow in scramjet from 1997. The group began to develop a parallel three dimensional CFD platform which is now called AHL3D at 2001, concerning of the complex configuration, different fuels (mainly $\mathrm{H} 2$ and hydrocarbons), chemically reacting flows, and computational efficiency(Yang Shunhua. 2006)(Zhao Huiyong. 2005)(Wang Lan. 2007).

At present, the CFD platform AHL3D is parallelized using MPI for multi-processor machines, and can be used to solve the Reynolds-averaged conservation equations (in 2-D, axisymmetric, 3-D, or PNS form) with a cell-center finite volume method. AHL3D can be used for calorically perfect gas flows, flows of an arbitrary mixture of thermally perfect gases undergoing non-equilibrium chemical reactions, two phase flows, steady and unsteady flows, laminar and turbulent flows. The platform can use multiblock, patched multiblock, or overset grids. The solutions are advanced in time with a LU-SGS scheme. A dual-time step scheme is used for unsteady flows. Now the group has made new progresses on the unstructured grid method and parabolized NS equation solver(Wang Lan. 2007).

The present paper adopts AHL3D to compute the internal flow field. 10 species and 12 chemical reactions are included. The turbulence model of TNT k- $\omega$ proposed by J.C.Kok is used. Taking the local cooling channel corresponding to the outer edge of boundary layer of the scramjet combustion gas, the flow field properties such as static pressure, static temperature, total temperature, velocity and mass proportion of the combustion gas species from the corresponding locations can be inputs for the formula (5).The influence on the combustion gas flow field by the heat transfer from gas to the wall is neglected.

\section{Structural analysis of the scramjet model}

In the present study, the total strain of the scramjet structure is decomposed in an elastic strain ,a plastic strain and a thermal strain. The basic equations for the structural analysis follow as(Kuhl,D. 2002)

$\nabla \vec{\sigma}=0, \forall \vec{x} \in \Omega$

$\vec{\sigma}=\vec{\sigma}(\vec{\varepsilon}, \alpha, T), \vec{\varepsilon}=\vec{\varepsilon}^{e}+\vec{\varepsilon}^{p}+\vec{\varepsilon}^{T}$

The boundary condition for the symmetry is

$\vec{n} \cdot \vec{u}=0$

The boundary condition for the front end of the scramjet is

$\vec{u}=0$

The boundary condition for the gas side wall is

$\vec{n} \cdot \vec{\sigma} \cdot \vec{n}=p_{h} \quad \forall \vec{x} \in \Gamma_{h}$

The boundary condition for the coolant side wall is

$\vec{n} \cdot \vec{\sigma} \cdot \vec{n}=p_{c} \quad \forall \vec{x} \in \Gamma_{c}$

\section{Principal results and interpretation}

Since scramjet has the symmetrical structure circling the $\mathrm{z}$ axes, thermal evaluation is performed for half a engine. The corresponding operating condition for the scramjet is: Mach number at the entrance of the isolated section is 3.1 , the inflow of air is vitiated, the total pressure is $3.87 \mathrm{MPa}$, the total temperature is $1650 \mathrm{~K}$, the mass flow rate of air is $1.46 \mathrm{~kg} / \mathrm{s}$, the fuel/air ratio is 1.2 .The corresponding flight Mach number is 5.86 .

The cooling kerosene enters the rear of the engine structure and is routed through the structure to the front of the engine. It is assumed that the mass flow rate of kerosene at the entrance for each channel of the 82 cooling channels is equivalent. To ensure that the amount of the cooling fuel matches that of the combustion fuel, the mass flow rate in each cooling channel is $1.019 \mathrm{~g} / \mathrm{s}$. The fuel entrance temperature is $300 \mathrm{~K}$, the fuel entrance pressure is $7.0 \mathrm{MPa}$. 
Figure 5 is the temperature contour of a cooling channel cross section in the scramjet side wall(at a location where $\mathrm{x}=0.981 \mathrm{~m}$ and $\mathrm{y}=0.141 \mathrm{~m}$ along a cooling channel center line), the temperature difference within the cross section is $91 \mathrm{~K}$.Figure 6 is the gas side wall temperature distribution, the temperature is uneven, the higher temperature locates in the area where $\mathrm{x}=0.902 \mathrm{~m} \sim 1.04 \mathrm{~m}$ in the sidewall, the highest value is $1104 \mathrm{~K}$.Figure 7 is the heated kerosene temperature distribution, it is heated from $300 \mathrm{~K}$ to $943 \mathrm{~K}$, the kerosene temperature in side wall is the highest. Figure 8 is the heat flux distribution, it is also uneven, the highest heat flux is $0.699 \mathrm{MW} / \mathrm{m}^{2}$.

After the thermal evaluation of scramjet has been completed, thermal environmental data and pressure data along scramjet flow path or each cooling channel can be introduced into the thermal-stress finite element program. Thermal environment data and pressure data on each node must be ascertained during the use of the finite element program. Figure 9 is the local wall temperature distribution of scramjet. To compare the result of Figure 9 with that of Figure 6, it indicates that the temperature from the thermal evaluation is fairly consistent with that from the finite element program. Figure 10 is the local stress distribution of scramjet. Most regions remain elastic, whereas small area exceeds elastic limit, it goes into the plastic state, thus thermo-elasto-plasticity of material is needed to study in the future work. Figure 11 is the displacement distribution of scramjet, the maximum displacement is $17 \mathrm{~mm}$.

\section{Conclusions}

1)Three dimensional internal flow results of scramjet from AHL3D, three-dimensional thermal evaluation code for the regeneratively cooled scramjet and the thermal-stress finite element program are incorporated together to develop numerical model which can be capable of accomplishing the thermal-structural analysis of the regenerative cooled scramjet.

2)The temperature and heat flux distribution of the scramjet structure is very uneven.

3)Local plasticity occurs. Further investigation and verification is required for the scramjet structure analysis.

\section{References}

Hank J M, Murphy J S and Mutzman R C. The X-51A scramjet engine flight demonstration program. AIAA 2008-2540.

HEISER W H, PRATT D T. (1994). Hypersonic airbreathing propulsion. American Institute of Aeronautics and Astronautics Inc., Washington,DC,1994.

Kuhl,D. (2002). Thermomechanical Analysis and Optimization of Cryogenic Liquid Rocket Engines. Journal of Propulsion and Power, Vol. 18, No. 4, July-August 2002.

Linne D L, Meyer M L, Edwards T, and Eitman D A. Evaluation of Heat Transfer and Thermal Stability of Supercritical JP-7 Fuel. AIAA-97-3041.

Mark A S. Hydrocarbon scramjet engine technology flowpath component development. Pratt\&Whitney Space Propulsion.

NARAGHI M H N. (2002). A computer code for three dimensional rocket thermal evaluation, user manual for RTE2002 version 1[R]. Tara Technologies ,LLC.

TAO Wen-quan. (2001). Numerical heat transfer (2nd ed.), Xi'an Jiaotong University Press, Xi'an.

Wang Lan. (2007). Parallel numerical simulations of whole scramjet engine flowfields on unstructured grids[D].China Aerodynamic Research and Development Center Graduate School.

Warwick G. (2008). Hyper reality. Aviation Week \& Space Technology, Nov.3, 2008, pp.62-64.

Wishart D P, Fortin T B, Guinan D, and Modroukas D. Design, fabrication and testing of an actively cooled scramjet propulsion system[R]. AIAA 2003-0015.

Yang Shunhua. (2006). Numerical study of hydrocarbon fueled scramjets[D]. China Aerodynamic Research and Development Center Graduate School.

Yu Chang-min. (1982). Numerical analysis of heat conduction. Tsinghua University Press.

Zhao Huiyong. (2005). Parallel numerical study of whole scramjet engine[D]. China Aerodynamic Research and Development Center Graduate School. 


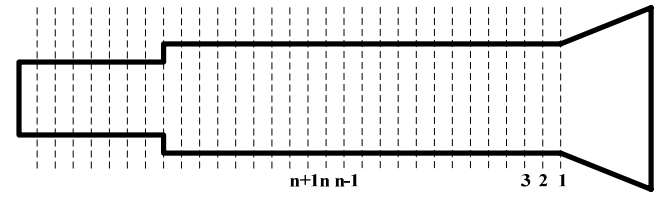

Figure 1. A scramjet model subdivided into a number of stations
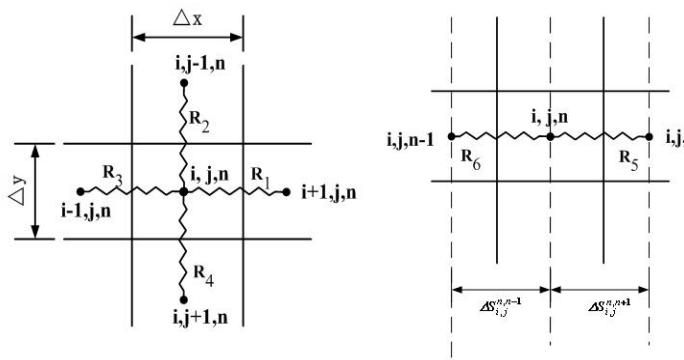

Figure 3. Resistances between a typical interior node and its adjoining nodes
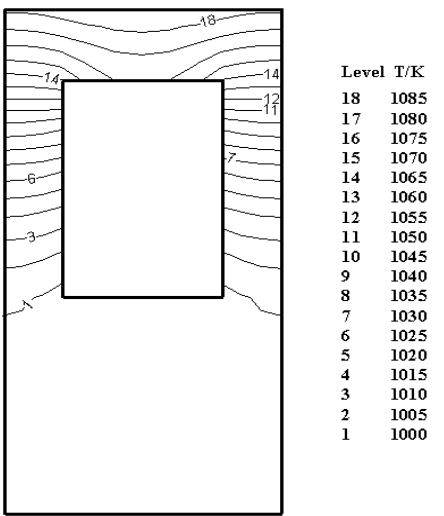

Figure 5. Temperature contour of a cooling channel cross section in scramjet side wall

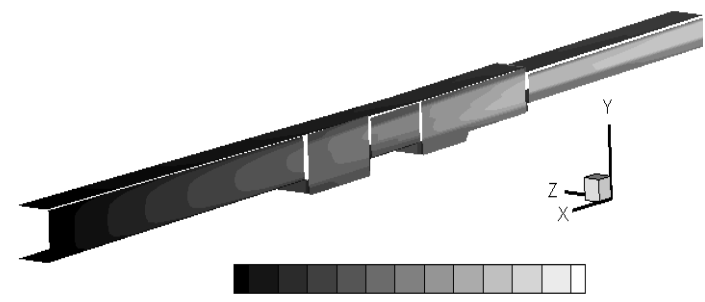

T1/K: 350400450500550600650700750800850900

Figure 7. Heated kerosene temperature distribution, $M a=5.86$

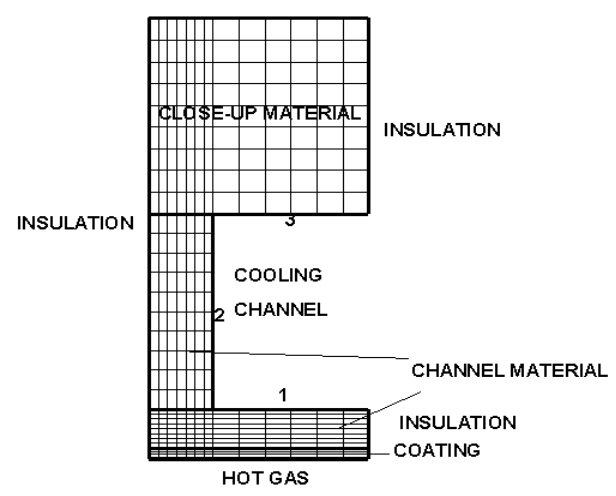

Figure 2. A half cooling channel cell and finite difference grid

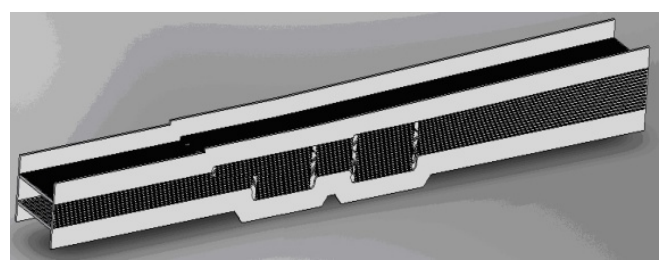

Figure 4. Scramjet model with cooling channels

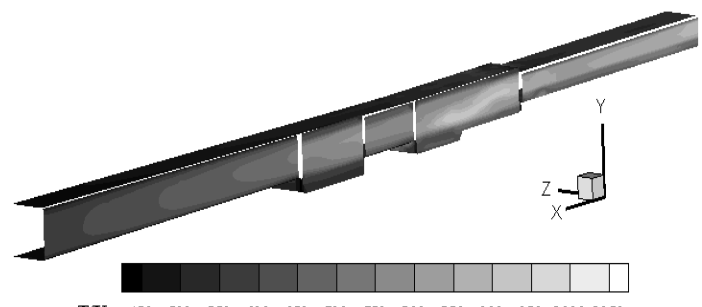

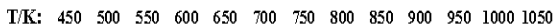

Figure 6. Gas side wall temperature distribution,

$$
M a=5.86
$$

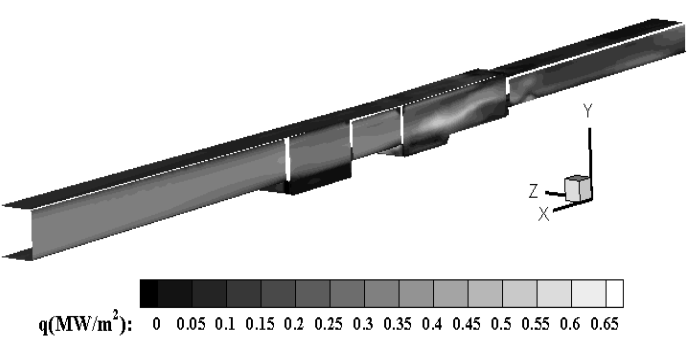

Figure 8. Heat flux distribution, $M a=5.86$ 


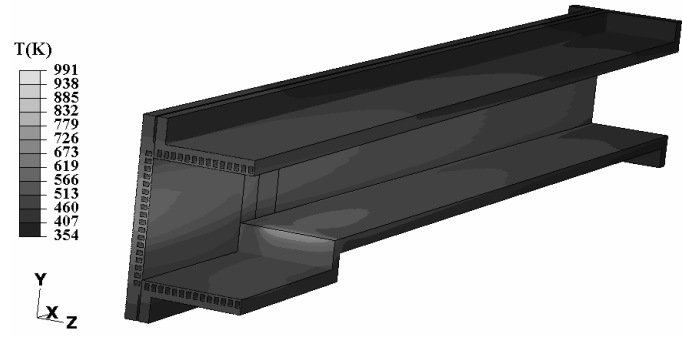

Figure 9. The local wall temperature distribution of scramjet

$U(m)$

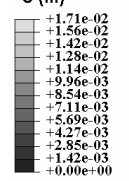

\begin{tabular}{r|r}
$\mathrm{Y}-\mathrm{z}$ \\
$\mathrm{y}$
\end{tabular}

Figure 11. The displacement distribution of scramjet

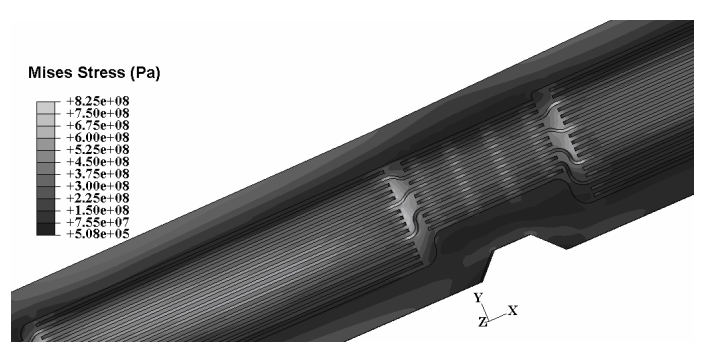

Figure 10. The local stress distribution of scramjet 